\title{
Electrically tunable detector of THz-frequency signals based on an antiferromagnet
}

\author{
Cite as: Appl. Phys. Lett. 117, 222411 (2020); doi: 10.1063/5.0031053 \\ Submitted: 27 September 2020 - Accepted: 25 November 2020 . \\ Published Online: 4 December 2020
}

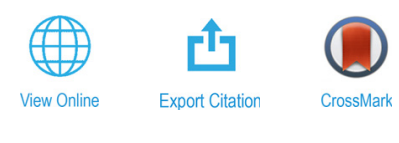

\begin{abstract}
A. Safin, ${ }^{1,2, a)}$ (D) V. Puliafito, ${ }^{3}$ (D) M. Carpentieri, ${ }^{4}$ (D) G. Finocchio, ${ }^{5}$ S. Nikitov, ${ }^{1,6,7}$ P. Stremoukhov, ${ }^{1,6,8}$ (iD A. Kirilyuk, ${ }^{1,8}$ (iD V. Tyberkevych, ${ }^{9}$ and A. Slavin ${ }^{9}$ (iD)
\end{abstract}

\author{
AFFILIATIONS \\ ${ }^{7}$ Kotel'nikov Institute of Radioengineering and Electronics, Russian Academy of Sciences, 125009 Moscow, Russia \\ ${ }^{2}$ National Research University “Moscow Power Engineering Institute," 111250 Moscow, Russia \\ ${ }^{3}$ Department of Engineering, University of Messina, I-98166 Messina, Italy \\ ${ }^{4}$ Department of Electrical and Information Engineering, Politecnico di Bari, I-70125 Bari, Italy \\ ${ }^{5}$ Department of Mathematical and Computer Sciences, Physical Sciences and Earth Sciences, University of Messina, \\ I-98166 Messina, Italy \\ ${ }^{6}$ Moscow Institute of Physics and Technology, Dolgoprudny, Moscow Region 141700, Russia \\ 7Laboratory “Metamaterials," Saratov State University, 83 Astrakhanskaya street, Saratov 410012, Russia \\ ${ }^{8}$ FELIX Laboratory, Radboud University, 6525 AJ Nijmegen, The Netherlands \\ ${ }^{9}$ Oakland University, Rochester, Michigan 48309, USA
}

a) Author to whom correspondence should be addressed: arsafin@gmail.com

\begin{abstract}
A concept of an electrically tunable resonance detector of THz-frequency signals based on an antiferromagnetic/heavy metal (AFM/HM) heterostructure is proposed. The conversion of a THz-frequency input signal into DC voltage is done using the inverse spin Hall effect in an (AFM/HM) bilayer. An additional bias DC in the HM layer can be used to vary the effective anisotropy of the AFM and, therefore, to tune the antiferromagnetic resonance (AFMR) frequency. The proposed AFM/HM heterostructure works as a resonance-type quadratic detector, which can be tuned by the bias current in the range of at least $10 \%$ of the AFMR frequency, and our estimations show that the sensitivity of this detector could be comparable to that of modern detectors based on the Schottky, Gunn, or graphene-based diodes.
\end{abstract}

Published under license by AIP Publishing. https://doi.org/10.1063/5.0031053

There is growing interest in the development of tunable oscillators and detectors operating in a terahertz $(\mathrm{THz})$ frequency range. Antiferromagnetic (AFM) materials have natural resonance frequencies of spin excitations (antiferromagnetic resonance or AFMR) lying in this frequency range. Some AFM materials can operate at room temperature, and they do not require any bias magnetic field and can be tuned by changing their anisotropy. These properties make AFM materials very attractive for use in $\mathrm{THz}$-frequency signal processing devices. The resonance detectors of terahertz $(\mathrm{THz})$ frequency signals have great potential for use in nondestructive testing, security, and telecommunication technologies ${ }^{1-4}$ since the $\mathrm{THz}$-frequency radiation has a relatively large penetration depth, being, at the same time, nonionizing. However, generation and resonance detection of signals with frequencies lying in the so-called "THz-gap" (from 0.1 to $10 \mathrm{THz}$ ) are rather difficult due to the rarity of naturally existing resonators operating in this frequency range. Vacuum electronic devices, semiconductors, and graphene-based oscillators with frequency multipliers can generate high-amplitude signals at frequencies of up to several hundreds of $\mathrm{GHz},{ }^{5}$ whereas light-based sources, such as quantum cascade lasers, provide signals with frequencies higher than several $\mathrm{THz}$ at room temperature, ${ }^{6}$ and so the THz-gap still exists. Some relief is provided by the oscillators and detectors based on Josephson junctions, ${ }^{7-10}$ but these devices require cryogenic temperatures for their operation, which creates a significant difficulty in their practical use. Thus, the development of small and simple room-temperature devices capable of generating and/or receiving resonant signals in a "THzgap" is still a significant challenge.

It was suggested previously to use AFM materials as active layers of $\mathrm{THz}$-frequency oscillators, due to the fact that the strong internal exchange magnetic field existing inside the AFM crystals pushes the 
frequencies of signals that can be generated in these crystals into the THz-frequency range. ${ }^{11}$ Also, there are several theoretical papers ${ }^{12-14}$ that suggested the possibility of development of nonresonant continuously tunable current-driven $\mathrm{THz}$-frequency auto-oscillators based on the effect of rotation of the AFM magnetic sublattices tilted by an external DC spin current in the large internal exchange magnetic field existing inside an AFM. It was predicted that the generation frequency of such AFM/heavy metal (HM) - based auto-oscillators, controlled by the DC bias electric current flowing in the HM layer, would vary between 0.1 and $2.0 \mathrm{THz}$ when the bias DC would be varied between $10^{8} \mathrm{~A} / \mathrm{cm}^{2}$ and $10^{9} \mathrm{~A} / \mathrm{cm}^{2}$.

Recently, ${ }^{15,16}$ it has been theoretically proposed to use active AFM generators for the detection of external THz-frequency signals via the mechanism of injection-locking of such a signal to the oscillations generated by a DC-driven $\mathrm{AFM} / \mathrm{HM} \mathrm{THz}$ generator.

An alternative way to develop quasi-passive AFM/HM-based detectors $^{17}$ is to use the fact that resonance eigenfrequencies of the AFM dynamic modes (standing AFMR modes) lie in the $\mathrm{THz}$ frequency range. It has been shown theoretically in Ref. 17 that a dielectric AFM having bi-axial anisotropy, such as $\mathrm{NiO}$, can be used for the resonance quadratic rectification of a linearly polarized AC spin current of $\mathrm{THz}$ frequency and could have a sensitivity in the range of $10^{2}-10^{3} \mathrm{~V} / \mathrm{W}$

The theoretical estimations of the AFM/HM detector parameters presented in Ref. 17 are rather encouraging, but for the practical use of such a detector, it is highly desirable to be able to continuously tune the resonance (AFMR) frequency of such a device by electric means, which was the main motivation of this work.

It should be noted that in the GHz frequency range, similar quadratic detectors based on the spin-torque magnetic diode (STMD) effect in ferromagnetic tunnel junctions has been investigated both theoretically and experimentally. ${ }^{18,19}$ The operating frequency of the ferromagnetic STMD is limited by the maximum possible applied bias magnetic field, and it is practically impossible to increase this frequency above several tens of GHz.

For the detector devices based on an AFM/HM heterostructure, the resonance (AFMR) frequencies are proportional to the square root of the product of the internal exchange and anisotropy magnetic fields [see Eq. (4)]. While the internal exchange magnetic field is very large (it reaches hundreds of Tesla) and fixed by the strong homogeneous exchange interaction, the AFM anisotropy field can be relatively easily controlled by various external means. In this work, we demonstrate a possibility to control the AFM anisotropy field and, therefore, the AFMR frequency, by changing a DC bias current in the HM layer of the AFM/HM heterostructure. To confirm our analytical results on the current-induced AFMR frequency tuning, we performed micromagnetic simulations by solving numerically the LandauLifthitz-Gilbert equation with a current-induced term (for more details, see our previous work ${ }^{20}$ ).

In this work, we consider a $\mathrm{THz}$ detector, schematically shown in Fig. 1, which consists of a uniaxial AFM driven by both DC (bias) and AC (signal) spin currents flowing from the bottom Pt layer. A spin current of the density $\boldsymbol{j}_{S H}$, produced due to the spin-orbit interaction in the bottom Pt layer, flows into the AFM and creates DC and AC spin torques with polarizations $\boldsymbol{n}_{\mathrm{DC}}$ and $\boldsymbol{n}_{\mathrm{AC}}$, respectively. These torques act on the magnetic sublattices. The precession of the magnetic sublattices due to the spin-pumping mechanism creates a spin current

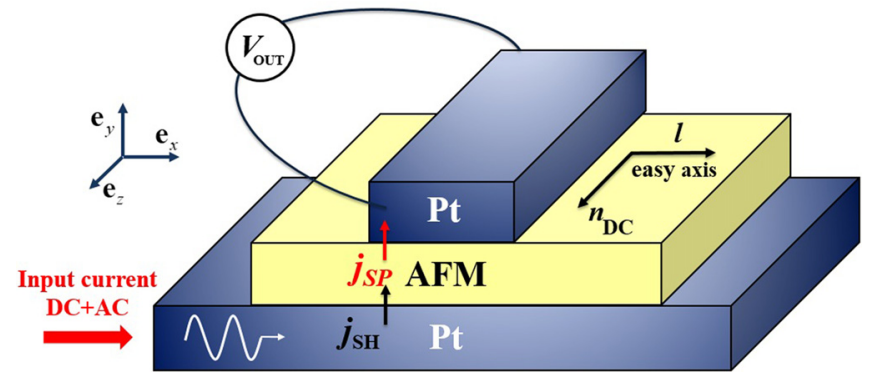

FIG. 1. Schematic view of the THz-frequency resonance detector based on the AFM-Pt structure, where $\boldsymbol{l}$ is the Néel vector oriented along the easy axis $\boldsymbol{n}_{\mathrm{e}}=\boldsymbol{e}_{\mathrm{x}}$ and $V_{\text {OUT }}$ is the output DC electric voltage. Due to the spin Hall effect, input electric current in Pt creates a spin current $\boldsymbol{j}_{S H}$, which has both DC and AC components. The polarization of the $A C$ spin current is directed perpendicular to the interface, while the polarization of the $D C p_{D C}=e_{z}$ is oriented in the interface plane and perpendicular to the easy axis. Oscillations of the Néel vector cause a spin current $j_{\mathrm{SP}}$ due to the spin-pumping mechanism. Both AC and DC spin-pumping signals are transformed into electric field signals via the inverse spin-Hall effect in the second Pt layer placed on top of the AFM.

$j_{\mathrm{SP}}$. This spin current via the inverse spin-Hall effect induces in the top Pt layer (see Fig. 1) an electric field in the direction $\boldsymbol{e}_{\mathrm{z}}$ perpendicular to the DC charge current flowing in the $\boldsymbol{e}_{\mathrm{x}}$-direction. We are interested in the DC part of the electric voltage $V_{\text {OUT }}$ induced in the top Pt layer between the output contacts that are separated by the distance $L=10 \mu \mathrm{m}$. Note that for the practical implementation of the proposed detector, one needs to measure a relatively small rectified DC voltage, proportional to the amplitude of the input AC signal, in the case when the bias DC could be rather large. Therefore, it is very important to guarantee that the source supplying the bias DC is highly stable.

Here, we consider a case when the polarization vector of $\boldsymbol{n}_{\mathrm{DC}}$ $=\boldsymbol{e}_{\mathrm{z}}$ of the DC is oriented perpendicular to the easy axis $\boldsymbol{e}_{\mathrm{e}}$ of the AFM anisotropy and the input AC spin current has a circular polarization described by the vector $\boldsymbol{n}_{\mathrm{AC}}=\left(\boldsymbol{e}_{\mathrm{y}} \pm i \boldsymbol{e}_{\mathrm{z}}\right) / \sqrt{2}$, where two signs correspond to the clockwise and anticlockwise rotation of the AC spin current polarization in the plane perpendicular to the easy axis $\boldsymbol{e}_{\mathrm{e}}$ of the AFM anisotropy. It should be mentioned that, as it was shown in Ref. 17, a linearly polarized AC spin current induces a zero output DC voltage in a uniaxial AFM, and so the circular polarization of the input AC is critical. An input AC having circular polarization could, for example, originate from a THz-frequency signal source placed in an EM resonator with a circular polarized magnetic field, ${ }^{23}$ could be obtained using an additional magnetic layer, which creates spin current with circular polarization in the z-y plane, or could be supplied by any other AFM-based THz-frequency oscillator. ${ }^{12,24}$ Previously, it was shown $^{25,26}$ that it is possible to rectify sub-terahertz electromagnetic waves via the inverse spin-Hall effect from uniaxial AFM perpendicular to the plane anisotropy axis. The excitation of the circularpolarized magnetic field was realized using coplanar waveguides. In this work, we consider the in-plane anisotropy axis.

We describe the AFM magnetization dynamics using the Néel vector $\boldsymbol{l}=\left(\boldsymbol{M}_{1}-\boldsymbol{M}_{2}\right) / 2 \boldsymbol{M}_{s}$, where $\boldsymbol{M}_{1,2}$ are the magnetization vectors of the AFM sublattices and $M_{\mathrm{S}}$ is the saturation magnetization of the sublattices (in particular, $M_{\mathrm{s}}=350 \mathrm{kA} / \mathrm{m}$ for IrMn at room temperature). The dynamics of the Néel vector $\boldsymbol{l}(t)$ is governed by the well-known equation of the so-called "sigma-model,"17,27-29 


$$
\boldsymbol{l} \times\left[\frac{1}{\omega_{\mathrm{ex}}} \frac{d^{2} \boldsymbol{l}}{d t^{2}}+\alpha_{\mathrm{eff}} \frac{d \boldsymbol{l}}{d t}+\hat{\Omega} \cdot \boldsymbol{l}+[\boldsymbol{\tau} \times \boldsymbol{l}]\right]=0 .
$$

Here, $\alpha_{\text {eff }}$ is the effective Gilbert damping constant, $\boldsymbol{e}_{\mathrm{e}}=\boldsymbol{e}_{\mathrm{x}}$ is the easy axis of the AFM anisotropy, $\hat{\Omega}=-\omega_{\mathrm{e}} \boldsymbol{e}_{\mathrm{e}} \otimes \boldsymbol{e}_{\mathrm{e}}$ and $\tau=\left(\omega_{\mathrm{DC}} \boldsymbol{n}_{\mathrm{DC}}\right.$ $+\omega_{\mathrm{AC}} \boldsymbol{n}_{\mathrm{AC}} e^{i \omega t}+$ c.c. $)$ is the DC and AC spin-transfer torque intensity. Characteristic frequencies are defined as follows:

$$
\left(\begin{array}{c}
\omega_{\mathrm{DC}} \\
\omega_{\mathrm{AC}}
\end{array}\right)=\sigma \cdot\left(\begin{array}{c}
j_{\mathrm{DC}} \\
j_{\mathrm{AC}}
\end{array}\right), \quad\left(\begin{array}{c}
\omega_{\mathrm{ex}} \\
\omega_{\mathrm{e}}
\end{array}\right)=\gamma \cdot\left(\begin{array}{c}
H_{\mathrm{ex}} \\
H_{\mathrm{e}}
\end{array}\right),
$$

where $j_{\mathrm{DC}}$ and $j_{\mathrm{AC}}$ are the densities of the input DC electric current and the AC electric current having frequency $\omega$, respectively, $\gamma$ is the modulus of the gyromagnetic ratio, $H_{\mathrm{ex}}$ is the AFM internal exchange magnetic field, and $H_{\mathrm{e}}$ is the AFM anisotropy field. The torque-current proportionally coefficient $\sigma$ is determined by the following expression: ${ }^{17}$

$$
\sigma=\frac{e \gamma \theta_{\mathrm{SH}} g_{r} \rho \lambda_{\mathrm{Pt}}}{2 \pi M_{s} d_{\mathrm{AFM}}} \tanh \left(\frac{d_{\mathrm{Pt}}}{2 \lambda_{\mathrm{Pt}}}\right),
$$

where $g_{r}=6.9 \times 10^{18} \mathrm{~m}^{-2}$ is the spin-mixing conductance at the AFM-Pt interface, $d_{\mathrm{AFM}}$ is the thicknesses of the AFM layer, $\theta_{\mathrm{SH}}$ $=0.1$ is the spin-Hall angle in $\mathrm{Pt}, \rho=4.8 \times 10^{-7} \Omega \mathrm{m}$ is the electrical resistivity of the Pt layer, $\lambda_{\mathrm{Pt}}=7.3 \mathrm{~nm}$ is the spin-diffusion length in $\mathrm{Pt}$, and $d_{\mathrm{Pt}}=20 \mathrm{~nm}$ is the Pt thickness. In our numerical simulations, we used a typical value of the effective Gilbert damping constant for $\mathrm{IrMn}^{30,31} \alpha_{\mathrm{eff}}=0.005$, which gives the quality factor $Q$ of the AFMR resonance approximately equal to 7 .

The resonance frequency of the detector in the simplest case of a uniaxial easy-axis AFM can be calculated using the expression, ${ }^{12}$

$$
\omega_{\mathrm{AFMR}}=\sqrt{\omega_{\mathrm{ex}} \omega_{\mathrm{e}}},
$$

where $\omega_{\mathrm{ex}}$ is the exchange frequency and $\omega_{\mathrm{e}}$ is the anisotropy frequency. For the uniaxial IrMn, with $\omega_{\mathrm{ex}} / 2 \pi=12.9 \mathrm{THz}$ and $\omega_{\mathrm{e}} / 2 \pi=16 \mathrm{GHz}$, the AFMR frequency $\omega_{\mathrm{AFMR}} / 2 \pi=454 \mathrm{GHz}^{30}$

We can describe the small-amplitude dynamics of the Néel vector as $\boldsymbol{l}=\boldsymbol{\lambda}+\boldsymbol{s} e^{i \omega t}+$ c.c., where $\boldsymbol{\lambda}$ is the ground state of the Néel vector, while vector $s$ describes the excitation created by the external AC spin current. These vectors satisfy the orthogonality condition $(\boldsymbol{\lambda} \cdot \boldsymbol{s})=0$.

Let us first consider the situation when the bias DC is absent. The ground state orientation of the Néel vector for the zero input DC density is $\lambda=(1,0,0)$ [see Fig. 2(a)], and so vector $\boldsymbol{\lambda}$ is oriented along the easy axis. The oscillations of the dynamic vector $s$ occur in the $\left(\boldsymbol{e}_{\mathrm{z}, \mathrm{y}}\right)$ plane, and the projection $s_{x}=0$. In this case, after simplifications, we can find an expression for the vector $s$ in the following form:

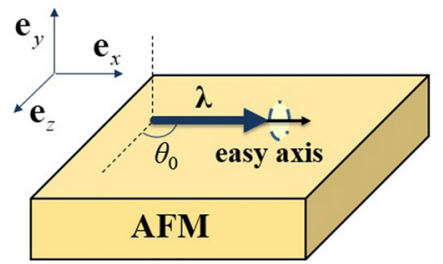

(a)

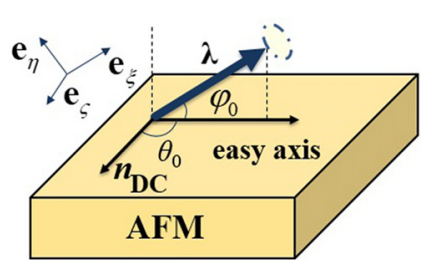

(b)
FIG. 2. Schematic representation of a Néel's ground state vector $\lambda$ orientation in an AFM for $j_{D C}=0$ (a) and for $j_{D C} \neq 0$ (b).

$$
\boldsymbol{s}=\frac{\omega_{\mathrm{AC}} \omega_{\mathrm{ex}}}{\omega_{A F M R}^{2}-\omega^{2}+i \gamma_{0} \omega} \cdot \boldsymbol{n}_{\mathrm{AC}},
$$

where $\gamma_{0}=\alpha_{\mathrm{eff}} \omega_{\mathrm{ex}}$ is the spectral linewidth of the AFMR for the zero input DC.

The rectified output DC spin current in the top Pt layer (see Fig. 1) is proportional to $\boldsymbol{j}_{\text {out }} \sim[\boldsymbol{l} \times \boldsymbol{i}] .^{12,17}$ From Eq. (5), after simplifications, one can find the output DC electric voltage between output contacts in the following form: ${ }^{12}$

$$
V_{\text {out }}(\omega)=\frac{V_{\max }^{0}\left(\gamma_{0}\right)^{2} \omega_{A F M R} \omega}{\left(\omega_{A F M R}^{2}-\omega^{2}\right)^{2}+\left(\gamma_{0} \omega\right)^{2}} .
$$

Here, $V_{\max }^{0}=\left(\frac{\omega_{A C}}{\gamma_{0}}\right)^{2} V_{0}$ is the maximum output DC voltage in the resonance case $\omega=\omega_{A F M R}$ and the normalized voltage $V_{0}$ is defined by the expression, ${ }^{17}$

$$
V_{0}=L e \omega_{\mathrm{ex}}^{2} \theta_{\mathrm{SH}} g_{r} \rho \lambda_{\mathrm{Pt}} \frac{\tanh \left(d_{\mathrm{Pt}} / 2 \lambda_{\mathrm{Pt}}\right)}{\pi d_{\mathrm{Pt}} \omega_{\mathrm{AFMR}}} .
$$

In our calculations, we assume that the AFM layer has a square cross section with the in-plane dimensions $S=100 \times 100 \mathrm{~nm}^{2}$ and the thickness $d_{\mathrm{AFM}}$ is $5 \mathrm{~nm}$. For $j_{\mathrm{AC}}=10^{7} \mathrm{~A} / \mathrm{cm}^{2}$, the output electric voltage is $V_{\text {out }} \approx 100 \mu \mathrm{V}$ at the zero DC input current density.

Figure 3 shows the standard resonance-type dependence of the output voltage $V_{\text {out }}$ on the frequency $\omega$ of the input AC signal. As it can be seen from Fig. 3, the spectral linewidth of the output voltage is equal to the linewidth of the AFM resonance $\gamma_{0} / 2 \pi=64.8 \mathrm{GHz}$.

Now, let us consider the case when a nonzero bias input DC is applied to the detector, as shown in Fig. 1. In this case [see Fig. 2(b)], the static equation defining the ground state Neel vector $\lambda$ can be found from (1) and has the following form:

$$
\boldsymbol{\lambda} \times(\hat{\boldsymbol{\Omega}} \cdot \boldsymbol{\lambda})+\omega_{\mathrm{DC}} \boldsymbol{\lambda} \times \boldsymbol{n}_{\mathrm{DC}} \times \boldsymbol{\lambda}=0 .
$$

Using a spherical coordinate system, one can express the ground state of the Néel vector as $\lambda=\left(\cos \left(\varphi_{0}\right) \sin \left(\theta_{0}\right), \sin \left(\varphi_{0}\right) \sin \left(\theta_{0}\right)\right.$, $\left.\cos \left(\theta_{0}\right)\right)$. It also follows from (1) that for $\boldsymbol{n}_{\mathrm{DC}}=\boldsymbol{e}_{\mathrm{z}}$ and $\boldsymbol{n}_{\mathrm{e}}=\boldsymbol{e}_{\mathrm{x}}$, the

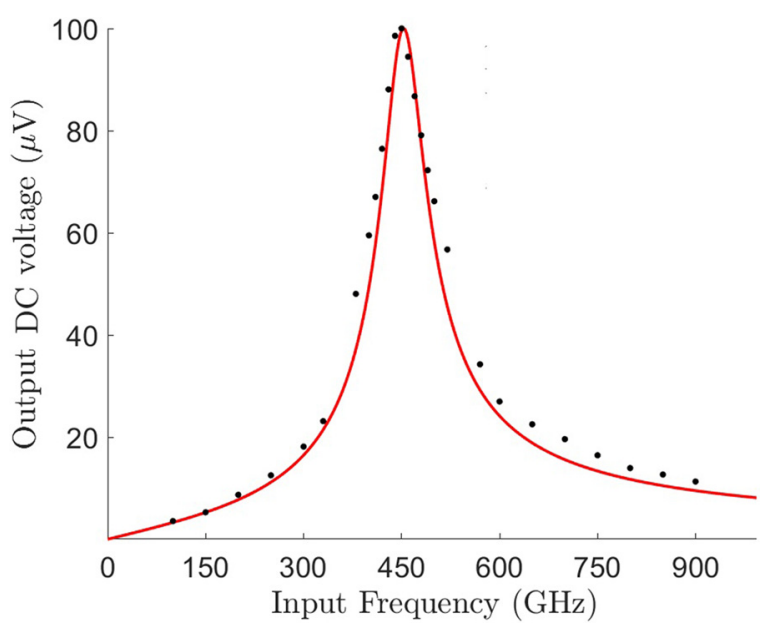

FIG. 3. Resonance curve of the output rectified DC voltage $V_{\text {out }}$ of an AFM-based detector calculated using Eq. (6) (solid line). Dots show the results of micromagnetic simulations. 
azimuthal angle $\theta_{0} \approx \pi / 2$ and the static polar angle of the Neel vector $\lambda$ is

$$
\varphi_{0}=\frac{1}{2} \arcsin \left(\frac{2 \omega_{\mathrm{DC}}}{\omega_{\mathrm{e}}}\right) .
$$

The increase in the polar angle $\varphi_{0}$ means that the static part of the Néel vector is deflected from the plane of the AFM interface, which results from the spin-transfer torque induced by the spins injected from the bottom Pt layer traversed by the external bias DC.

The "dynamic" equations defining the "excitation" vector $\boldsymbol{s}$, after some simplifications, can be written in the following form:

$$
\begin{aligned}
& \left(-\frac{\omega^{2}}{\omega_{\mathrm{ex}}}+i \omega \alpha_{\mathrm{eff}}\right) \boldsymbol{s}+(\hat{\boldsymbol{\Omega}}-(\boldsymbol{\lambda} \cdot(\hat{\boldsymbol{\Omega}} \cdot \boldsymbol{\lambda})) \hat{I}) \cdot \boldsymbol{s} \\
& \quad-(\boldsymbol{\lambda} \cdot(\hat{\boldsymbol{\Omega}} \cdot \boldsymbol{s})) \boldsymbol{\lambda}+\omega_{\mathrm{DC}}\left(\boldsymbol{\lambda} \cdot \boldsymbol{n}_{\mathrm{DC}}\right) \boldsymbol{\lambda} \times \boldsymbol{s}=\omega_{\mathrm{AC}} \boldsymbol{\lambda} \times \boldsymbol{n}_{\mathrm{AC}} .
\end{aligned}
$$

As it was mentioned earlier, for the zero input bias DC, the oscillations of the vector $s$ take place in the $(z, y)$ plane and $s_{x}=0$, whereas in the presence of the DC bias current, it is necessary to introduce a new coordinate system $\left(\boldsymbol{e}_{\xi}, \boldsymbol{e}_{\eta}, \boldsymbol{e}_{\zeta}\right)$ [see Fig. 2(b)], where the component $s_{\xi}$ perpendicular to the plane of oscillation $\left(\boldsymbol{e}_{\eta}, \boldsymbol{e}_{\zeta}\right)$ is equal to zero. The expressions for the components $s_{\eta}, s_{\zeta}$ are equivalent to those for the components $s_{y}, s_{z}$ (5) if we replace $\boldsymbol{e}_{y}$ by $\boldsymbol{e}_{\eta}, \boldsymbol{e}_{z}$ by $\boldsymbol{e}_{\zeta}$ and $\omega_{\text {AFMR }}$ by $\omega_{0}=\omega_{\text {AFMR }} \sqrt{\cos \left(2 \varphi_{0}\right)}$, respectively.

Recently, ${ }^{32}$ for the case of a biaxial AFM (e.g., NiO), it has been shown that in $\mathrm{NiO}$, there are two resonance frequencies $\omega_{1,2}$, and they are substantially different due to the strong difference between the anisotropies corresponding to the "easy" and "hard" axes. In the case of a uniaxial AFM, two AFMR eigenfrequencies are degenerate for the zero DC bias current. Although for a nonzero DC bias current, the dependencies $\omega_{1,2}\left(j_{\text {DC }}\right)$ differ, the difference is rather small, and in a uniaxial case, we still can approximately assume that $\omega_{1} \approx \omega_{2}=\omega_{0}$. The dependence of the resonance oscillation frequency on the DC bias current density in a uniaxial AFM can be expressed as

$$
\omega_{0}=\omega_{\mathrm{AFMR}} \sqrt[4]{1-\left(\frac{2 \omega_{\mathrm{DC}}}{\omega_{\mathrm{e}}}\right)^{2}} .
$$

The oscillation frequency (11) is proportional to the AFMR frequency $\omega_{\mathrm{AFMR}}$ and depends on the density $j_{\mathrm{DC}}$ of the DC bias current. Thus, the resonance frequency of magnetization oscillations in a uniaxial AFM can be tuned (reduced) by the variation of the bias DC density $j_{\mathrm{DC}}$ in the bottom Pt layer of the detector, as shown in Fig. 1.

Figure 4(a) shows the dependence of the oscillation frequency $\omega_{0}$ Eq. (11) as a function of the DC density in the sub-critical (passive) regime. It is clear that the frequency $\omega_{0}$ can be continuously reduced from $\omega_{\mathrm{AFMR}}$ by at least $10 \%$ through the increase in the bias current density to $5 \times 10^{8} \mathrm{~A} / \mathrm{cm}^{2}$.

The threshold current $j_{\text {th }}$ at which the detector in Fig. 1 enters the auto-oscillation (super-critical or active) regime can be easily found from the stability analysis of the damped oscillation mode in Eq. (1) in the form $j_{\text {th }}=\omega_{e} /(2 \sigma) \approx 7 \times 10^{8} \mathrm{~A} / \mathrm{cm}^{2}{ }^{12}$ This value of the current density is rather high. Note, however, that both the AFMR frequency and the threshold DC density corresponding to the transfer to the auto-oscillation regime can be substantially reduced in PZ/ AFM/Pt heterostructures ${ }^{32}$ based on a thin dielectric AFM layer using the magneto-elastic interaction for the voltage control of the AFM anisotropy.
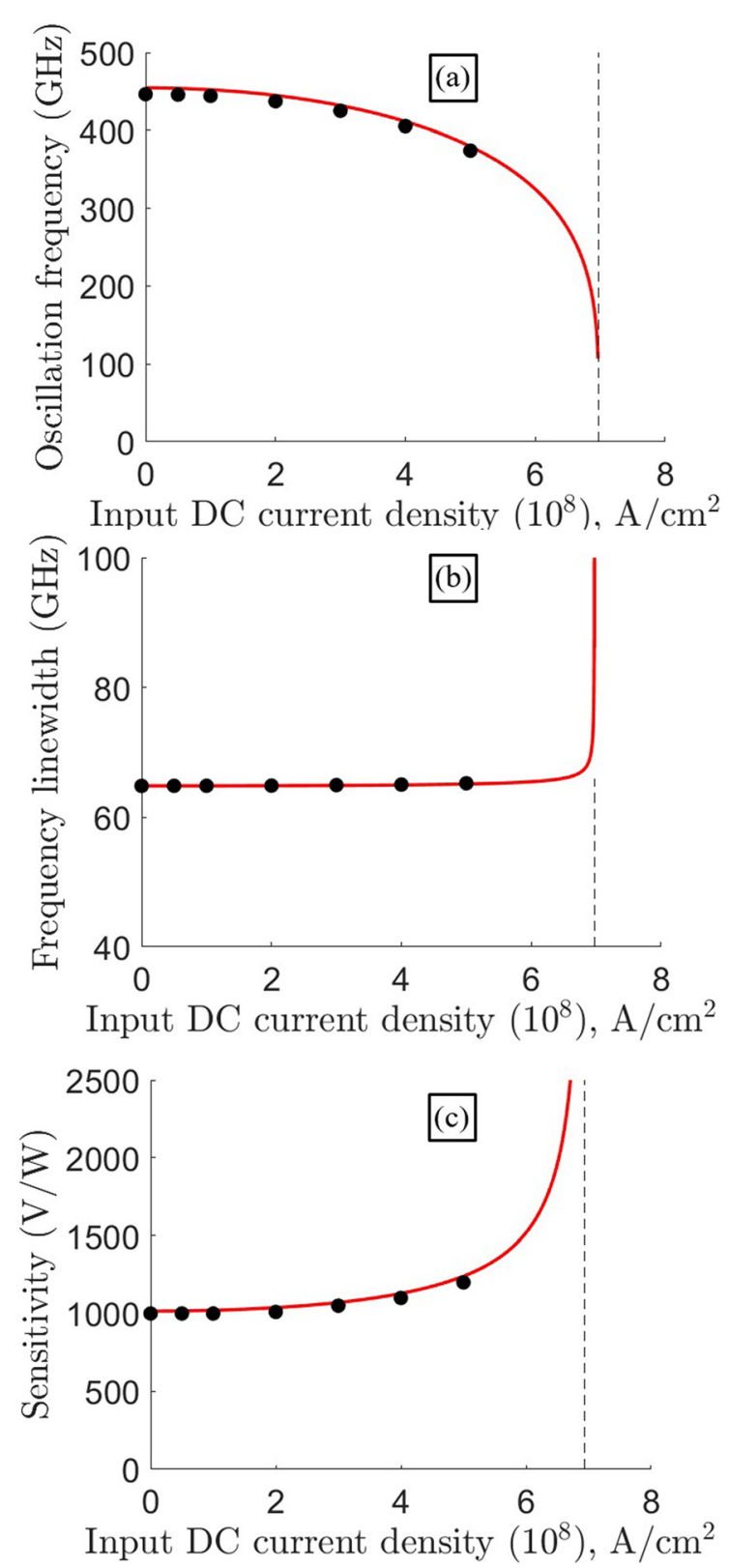

FIG. 4. Comparison between the results of analytical calculations (solid line) and micromagnetic simulations (dots), showing the dependence of the AFMR oscillation frequency (11) (a), frequency bandwidth (12) (b), and detector sensitivity (13) (c) on the input bias DC density flowing in the bottom Pt layer of the detector, shown in Fig. 1. Dashed lines show the dependences of the oscillation frequency, spectral linewidth, and sensitivity in the strongly nonlinear regime near the threshold of the auto-oscillation (super-critical) regime. Dots show the results of micromagnetic simulations.

It can be shown from (5) that the dependence $\Delta \omega\left(j_{\mathrm{DC}}\right)$ for sufficiently low DC densities can be found as follows:

$$
\Delta \omega=\gamma_{0}\left(1+\frac{\alpha_{\mathrm{eff}}^{2} \omega_{\mathrm{ex}}}{2 \omega_{\mathrm{e}}} \cdot\left(\frac{\omega_{\mathrm{DC}}}{\omega_{\mathrm{e}}}\right)^{2}\right) .
$$


Figure 4(b) shows the dependence of the frequency linewidth $\Delta \omega$ on the input bias DC density. As it can be seen from Fig. 4(b), the linewidth in the sub-critical (passive) regime is practically independent of the DC bias current. Of course, for the larger DC bias current densities, close to the auto-oscillation threshold, formula (12) is incorrect, and a more accurate theoretical analysis is necessary. The linewidth $64.8 \mathrm{GHz}$ achieved at the zero DC density corresponds to the quality factor $Q=\omega_{0} / \Delta \omega \approx 7$. Although this $Q$-factor is relatively small, it is still sufficient for the resonance reception of THz-frequency $\mathrm{AC}$ signals.

Finally, we calculate the detector sensitivity $\mathscr{R}=V_{\max } / P_{\mathrm{AC}}$, where $V_{\max }$ is the maximum rectified DC voltage $V_{\max }=V_{\max }^{0} \cdot \frac{\cos \left(\varphi_{0}\right)}{\sqrt{\cos \left(2 \varphi_{0}\right)}}$ for the nonzero input bias $\mathrm{DC}$ and $P_{\mathrm{AC}}=\rho j_{\mathrm{AC}}^{2} S d_{\mathrm{AFM}}$ is the input $\mathrm{AC}$ electric power. For $j_{\mathrm{AC}}=10^{7} \mathrm{~A} / \mathrm{cm}^{2}$, the input $\mathrm{AC}$ power is $P_{\mathrm{AC}} \approx 100$ nW. The output signal (see Fig. 1) from the Pt layer can be detected via the ISHE in the symmetric Pt/AFM/Pt structure (see, for more details, e.g., Refs. 21 and 22).

At the signal frequencies close to the AFMR, the detector sensitivity $\mathscr{R}_{0}$ exceeds $1000 \mathrm{~V} / \mathrm{W}$. Figure 4 (c) shows the dependence of the resonance sensitivity $\mathscr{R}$ on the input DC bias current density, which is calculated using Eq. (6) for $\omega=\omega_{0}$ and relatively small DC bias current densities in the following form:

$$
\mathscr{R}=\mathscr{R}_{0} \cdot\left(1+\frac{1}{2} \cdot\left(\frac{\omega_{\mathrm{DC}}}{\omega_{\mathrm{e}}}\right)^{4}\right) .
$$

Here, $\mathscr{R}_{0}=\left(\frac{\sigma}{\gamma_{0}}\right)^{2} \frac{V_{0}}{\rho S d_{\mathrm{AFM}}}$ is the sensitivity for the zero DC bias current density. As it can be seen from (13), the sensitivity increases slightly with the increase in the input DC bias current density. Note that for more accurate estimation of the device sensitivity at room temperature, the thermal fluctuations in the AFM material should be taken into account (following, e.g., Ref. 33), which we are planning to do in one of our subsequent works.

In conclusion, we have demonstrated theoretically that an AFM having uniaxial anisotropy can be used as a sensitive element for the resonance detection of $\mathrm{THz}$-frequency spin currents. We have shown that an additional bias DC in the HM layer can be used to reduce the effective anisotropy of the AFM layer and, therefore, to continuously tune the AFM resonance frequency. Analogous calculations can be made for a biaxial AFM ( $\mathrm{NiO}$, hematite $\alpha-\mathrm{Fe}_{2} \mathrm{O}_{3}$, etc.) as well. The proposed $\mathrm{AFM} / \mathrm{HM}$ heterostructure works as a resonance-type quadratic detector, which can be tuned by a bias DC in the range of at least $10 \%$ of the AFMR frequency. We have shown that for the zero input DC, a circularly polarized external AC excites the rotation of the Neel vector in a plane perpendicular to the interface. Practical realization of circularly polarization of the AC is the subject of a separate study, and it will be considered in our future works. Our estimations also show that the sensitivity of the proposed AFM detector of THzfrequency signals could be comparable to that of modern detectors based on the Schottky, Gunn, or graphene-based diodes ${ }^{5}$ (with the maximum sensitivity of the order of $10^{5} \mathrm{~V} / \mathrm{W}$ ). We anticipate that this described detector effect can be observed experimentally using the electric injection and detection of spin currents via the spin Hall effects in HM/AFM bilayers, like in the pioneering experiments shown in Refs. 34 and 35. In our opinion, the proposed AFM-based resonance detectors of THz-frequency signals can be used as general-purpose receivers of $\mathrm{THz}$-frequency radiation, as sensors for the detection of THz-frequency spin currents generated by ultra-fast AFM artificial neurons in neuromorphic computational architectures, ${ }^{36,37}$ and as sensitive elements in THz-frequency spectrum analyzers ${ }^{38}$ and CMOScompatible AFM-based memory cells. ${ }^{39}$

This work was supported in part by the U.S. National Science Foundation (Grant No. EFMA-1641989), by the U.S. Air Force Office of Scientific Research under MURI Grant No. FA9550-19-10307, and by the Oakland University Foundation.

The work of V.P., M.C., and G.F. was supported under Grant No. 2019-1-U.0. ("Diodi spintronici rad-hard ad elevata sensitivitáDIOSPIN") funded by the Italian Space Agency (ASI) within the call "Nuove idee per la componentistica spaziale del futuro."

We would like to acknowledge networking support from COST Action No. CA17123 "Ultrafast opto magneto electronics for nondissipative information technology."

Studies of the detection were carried out with the support of the Russian Science Foundation (Grant No. 19-19-00607) and the grant from the Government of the Russian Federation for state support of scientific research conducted under the guidance of leading scientists in Russian higher education institutions, research institutions, and state research centers of the Russian Federation (Project No. 075-15-2019-1874). Studies of the magnetic dynamics in antiferromagnetics were supported by the RFBR under Grant Nos. 18-37-20048, 18-29-27018, 18-57-76001, 18-07-00509A, and 18-29-27020 and by the grants of the President of the Russian Federation (Nos. MK-283.2019.8 and MK-3607.2019.9). S.A. acknowledges support from the Government of the Russian Federation (Grant No. 074-02-2018-286 for the "Terahertz Spintronics" laboratory of the MIPT). A.R. acknowledges the support from the RFBR (Grant No. 19-29-03015). P.A. acknowledges the support from the RFBR (Grant No. 19-32-90242).

\section{DATA AVAILABILITY}

The data that support the findings of this study are available from the corresponding author upon reasonable request.

\section{REFERENCES}

${ }^{1}$ A. Y. Pawar, D. D. Sonawane, K. B. Erande, and D. V. Derle, "Terahertz technology and its applications,” Drug Invent. Today 5, 157-163 (2013).

${ }^{2}$ J. F. Federici, B. Schulkin, F. Huang, D. Gary, R. Barat, F. Oliveira, and D. Zimdars, "THz imaging and sensing for security applications-explosives, weapons and drugs," Semicond. Sci. Technol. 20, S266 (2005).

${ }^{3}$ F. Sizov and A. Rogalski, "THz detectors," Prog. Quantum Electron. 34, 278-347 (2010).

${ }^{4}$ B. Ferguson and X.-C. Zhang, "Materials for terahertz science and technology," Nat. Mater. 1, 26-33 (2002).

${ }^{5}$ R. A. Lewis, "A review of terahertz sources," J. Phys. D 47, 374001 (2014).

${ }^{6}$ B. S. Williams, "Terahertz quantum cascade lasers," in Asia Optical Fiber Communication and Optoelectronic Exposition and Conference (Optical Society of America, 2008), p. SuG3.

${ }^{7}$ L. Ozyuzer, A. E. Koshelev, C. Kurter, N. Gopalsami, Q. Li, M. Tachiki, K. Kadowaki, T. Yamamoto, H. Minami, H. Yamaguchi et al., "Emission of coherent THz radiation from superconductors," Science 318, 1291-1293 (2007).

${ }^{8} \mathrm{X}$. Hu and S.-Z. Lin, "Phase dynamics in a stack of inductively coupled intrinsic Josephson junctions and terahertz electromagnetic radiation," Supercond. Sci. Technol. 23, 053001 (2010).

${ }^{9}$ A. Barone and G. Paterno, Physics and Applications of the Josephson Effect (Wiley, 1982). 
${ }^{10} \mathrm{~K}$. K. Likharev, Dynamics of Josephson Junctions and Circuits (Gordon and Breach Science Publishers, 1986).

${ }^{n} \mathrm{R}$. Cheng, D. Xiao, and A. Brataas, "Terahertz antiferromagnetic spin Hall nano-oscillator," Phys. Rev. Lett. 116, 207603 (2016)

${ }^{12}$ R. Khymyn, I. Lisenkov, V. Tiberkevich, B. A. Ivanov, and A. Slavin, "Antiferromagnetic THz-frequency Josephson-like oscillator driven by spin current," Sci. Rep. 7, 43705 (2017).

${ }^{13}$ O. Sulymenko, O. Prokopenko, V. Tiberkevich, A. Slavin, B. Ivanov, and R. Khymyn, "Terahertz-frequency spin Hall auto-oscillator based on a canted antiferromagnet," Phys. Rev. Appl. 8, 064007 (2017).

${ }^{14}$ O. R. Sulymenko, O. V. Prokopenko, V. S. Tyberkevych, and A. N. Slavin, "Terahertz-frequency signal source based on an antiferromagnetic tunnel junction," IEEE Magn. Lett. 9, 1-5 (2018).

${ }^{15} \mathrm{O}$. Gomonay, T. Jungwirth, and J. Sinova, "Narrow-band tunable terahertz detector in antiferromagnets via staggered-field and antidamping torques," Phys. Rev. B 98, 104430 (2018).

${ }^{16} \mathrm{R}$. Khymyn, V. Tiberkevich, and A. Slavin, "Injection locking of the antiferromagnetic oscillator," in Book of Abstracts "Intermag" (2018), p. 795.

${ }^{17}$ R. Khymyn, V. Tiberkevich, and A. Slavin, "Antiferromagnetic spin current rectifier," AIP Adv. 7, 055931 (2017).

${ }^{18}$ A. A. Tulapurkar, Y. Suzuki, A. Fukushima, H. Kubota, H. Maehara, K. Tsunekawa, D. D. Djayaprawira, N. Watanabe, and S. Yuasa, "Spin-torque diode effect in magnetic tunnel junctions," Nature 438, 339-342 (2005).

${ }^{19}$ O. Prokopenko, G. Melkov, E. Bankowski, T. Meitzler, V. Tiberkevich, and A. Slavin, "Noise properties of a resonance-type spin-torque microwave detector," Appl. Phys. Lett. 99, 032507 (2011).

${ }^{20}$ V. Puliafito, R. Khymyn, M. Carpentieri, B. Azzerboni, V. Tiberkevich, A. Slavin, and G. Finocchio, "Micromagnetic modeling of terahertz oscillations in an antiferromagnetic material driven by the spin Hall effect," Phys. Rev. B 99, 024405 (2019).

${ }^{21}$ M. Yang, K. Cai, H. Ju, K. W. Edmonds, G. Yang, S. Liu, B. Li, B. Zhang, Y. Sheng, S. Wang, Y. Ji, and K. Wang, "Spin-orbit torque in pt/CoNiCo/pt symmetric devices," Sci. Rep. 6, 20778 (2016).

${ }^{22}$ H. Wu, C. H. Wan, X. Zhang, Z. H. Yuan, Q. T. Zhang, J. Y. Qin, H. X. Wei, X. F. Han, and S. Zhang, "Observation of magnon-mediated electric current drag at room temperature," Phys. Rev. B 93, 060403 (2016).

${ }^{23}$ K. K. Jain and P. Kishan, "Microwave ferrite devices," IETE Tech. Rev. 14, 373-386 (1997).

${ }^{24}$ I. Lisenkov, R. Khymyn, J. Ackerman, N. Sun, and B. A. Ivanov, "Subterahertz ferrimagnetic spin-transfer torque oscillator," Phys. Rev. B 100, 100409(R) (2019).

${ }^{25}$ P. Ross, M. Schreier, J. Lotze, H. Huebl, R. Gross, and S. T. B. Goennenwein, "Antiferromagentic resonance detected by direct current voltages in $\mathrm{MnF}_{2} / \mathrm{pt}$ bilayers," J. Appl. Phys. 118, 233907 (2015).
${ }^{26}$ P. Vaidya, S. A. Morley, J. van Tol, Y. Liu, R. Cheng, A. Brataas, D. Lederman, and E. del Barco, "Subterahertz spin pumping from an insulating antiferromagnet," Science 368, 160-165 (2020).

${ }^{27}$ T. Satoh, S.-J. Cho, R. Iida, T. Shimura, K. Kuroda, H. Ueda, Y. Ueda, B. Ivanov, F. Nori, and M. Fiebig, "Spin oscillations in antiferromagnetic nio triggered by circularly polarized light," Phys. Rev. Lett. 105, 077402 (2010).

${ }^{28}$ R. Khymyn, I. Lisenkov, V. S. Tiberkevich, A. N. Slavin, and B. A. Ivanov, "Transformation of spin current by antiferromagnetic insulators," Phys. Rev. B 93, 224421 (2016)

${ }^{29}$ L. Sánchez-Tejerina, V. Puliafito, P. K. Amiri, M. Carpentieri, and G. Finocchio, "Dynamics of domain-wall motion driven by spin-orbit torque in antiferromagnets," Phys. Rev. B 101, 014433 (2020).

${ }^{30} \mathrm{O}$. V. Gomonay and V. M. Loktev, "Using generalized Landau-Lifshitz equations to describe the dynamics of multi-sublattice antiferromagnets induced by spin-polarized current," Low Temp. Phys. 41, 698-704 (2015).

${ }^{31}$ W. Zhang, Q. Liu, Z. Yuan, K. Xia, W. He, Q.-F. Zhan, X.-Q. Zhang, and Z.-H. Cheng, "Enhancement of ultrafast demagnetization rate and gilbert damping driven by femtosecond laser-induced spin currents in $\mathrm{Fe}_{81} \mathrm{Ga}_{19} / \mathrm{Ir}_{20} \mathrm{Mn}_{80}$ bilayers," Phys. Rev. B 100, 104412 (2019).

${ }^{32}$ P. Popov, A. Safin, A. Kirilyuk, S. Nikitov, I. Lisenkov, V. Tyberkevich, and A. Slavin, "Voltage-controlled anisotropy and current-induced magnetization dynamics in antiferromagnetic/piezoelectric heterostructures," Phys. Rev. Appl. 13, 044080 (2020).

${ }^{33}$ Y. G. Semenov, X. Xu, and K. W. Kim, "Thermal fluctuations in antiferromagnetic nanostructures,” J. Magn. Magn. Mater. 489, 165457 (2019).

${ }^{34}$ R. Lebrun, A. Ross, S. Bender, A. Qaiumzadeh, L. Baldrati, J. Cramer, A. Brataas, R. Duine, and M. Kläui, "Tunable long-distance spin transport in a crystalline antiferromagnetic iron oxide," Nature 561, 222-225 (2018).

${ }^{35} \mathrm{M}$. Tarequzzaman, A. S. Jenkins, T. Böhnert, J. Borme, L. Martins, E. Paz, R. Ferreira, and P. P. Freitas, "Broadband voltage rectifier induced by linear bias dependence in $\mathrm{CoFeB} / \mathrm{MgO}$ magnetic tunnel junctions," Appl. Phys. Lett. 112, 252401 (2018).

${ }^{36}$ R. Khymyn, I. Lisenkov, J. Voorheis, O. Sulymenko, O. Prokopenko, V. Tiberkevich, J. Akerman, and A. Slavin, "Ultra-fast artificial neuron: Generation of picosecond-duration spikes in a current-driven antiferromagnetic auto-oscillator," Sci. Rep. 8, 15727 (2018).

${ }^{37}$ O. Sulymenko, O. Prokopenko, I. Lisenkov, J. Ảkerman, V. Tyberkevych, A. N. Slavin, and R. Khymyn, "Ultra-fast logic devices using artificial "neurons" based on antiferromagnetic pulse generators," J. Appl. Phys. 124, 152115 (2018).

${ }^{38}$ P. Artemchuk, O. Sulymenko, S. Louis, R. Khymyn, E. Bankowski, T. Meitzler, V. Tyberkevich, A. Slavin, and O. Prokopenko, "Terahertz frequency spectrum analysis with a nanoscale antiferromagnetic tunnel junction," J. Appl. Phys. 127, $063905(2020)$

${ }^{39}$ J. Shi, V. Lopez-Dominguez, F. Garesci, C. Wang, H. Almasi, M. Grayson, G. Finocchio, and P. K. Amiri, "Electrical manipulation of the magnetic order in antiferromagnetic PtMn pillars," Nat. Electron. 3, 92-98 (2020). 